\title{
Sensory systems processing in children with spastic cerebral palsy: a pilot study
}

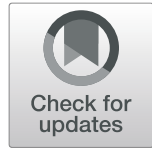

Mennatallah Khamis AbdelHafiez Barakat ${ }^{*}$ id, Gehan Hassan Elmeniawy and Faten Hassan Abdelazeim

\begin{abstract}
Background: The study of the sensory aspect alongside the motor aspect in children with spastic cerebral palsy is critical. Knowledge may help improve intervention, children's quality of life, activity, and participation.

The study aims to analyze the sensory systems processing in children with spastic cerebral palsy classified by Gross Motor Function Classification System (GMFCS) as levels I, II, and III using Child Sensory Profile 2 (CSP-2). Caregivers of children with spastic cerebral palsy aged 6-9 years $(n=20)$ completed the CSP-2. Children with severe comorbidities besides cerebral palsy (i.e., diabetes, myopathy, neuropathy, and uncontrolled epilepsy), autism, burn injuries, hearing or vision loss were excluded.

Results: All twenty participating children showed sensory processing difficulties in at least one of the sensory sections. With 14 out of 20 showing abnormalities in more than one section, the majority showed problems in the body position section, followed by the movement section. There was a significant difference between raw scores means of children with unilateral and bilateral SCP at the "body position" section, unlike the rest of the other sections.

Conclusions: Children with spastic cerebral palsy (GMFCS levels I, II, and III) show sensory systems processing difficulties compared to normative data, especially at vestibular and proprioceptive processing.
\end{abstract}

Keywords: Cerebral palsy, Sensory Processing, Sensory Profile 2, Sensory Processing Disorder

\section{Background}

Cerebral palsy $(\mathrm{CP})$ is a neurodevelopmental disorder that affects muscle tone, movement, and motor skills. Clinical signs are persistent and non-progressive, resulting from a lesion to the developing brain in the prenatal, perinatal, or early postnatal period $[1,2]$.

Spastic cerebral palsy (SCP) is the most common type of CP, composing up to $70 \%$ to $80 \%$ of cases [3]. SCP may be bilateral or unilateral [4].

Motor abnormalities are frequently accompanied by sensory, perceptual, cognitive, communication, and behavior disturbances, as well as epilepsy and secondary musculoskeletal problems [5]. Therefore, the management of children with CP depends on a multidisciplinary, comprehensive, and coordinated approach and goals are

\footnotetext{
* Correspondence: mennakhamis@hotmail.com

Department of Physical Therapy for Growth and Development Disorders in Children and Its Surgery, Faculty of Physical Therapy, Cairo University, Giza, Egypt
}

(c) The Author(s). 2021 Open Access This article is licensed under a Creative Commons Attribution 4.0 International License, which permits use, sharing, adaptation, distribution and reproduction in any medium or format, as long as you give appropriate credit to the original author(s) and the source, provide a link to the Creative Commons licence, and indicate if changes were made. The images or other third party material in this article are included in the article's Creative Commons licence, unless indicated otherwise in a credit line to the material. If material is not included in the article's Creative Commons licence and your intended use is not permitted by statutory regulation or exceeds the permitted use, you will need to obtain permission directly from the copyright holder. To view a copy of this licence, visit http://creativecommons.org/licenses/by/4.0/.

the child's independence and being engaged in the community [3].

Sensory processing is the processing of sensory information from the environment and the body itself by neural systems, comprising the functions of receptor organs and the peripheral and central nervous systems. To understand experiences and organize responses, the brain organizes, integrates, synthesizes, and uses this information. Individuals can respond to sensory stimuli in an automatic, efficient, and comfortable manner thanks to information processing [6].

The term sensory processing disorder (SPD) is used by the Sensory Processing Disorder Foundation to define a condition in which a person's daily activities are limited by how he or she processes and responds to sensory information [7]. SPD is characterized by hypo- or hypersensitivities to sensory inputs that cause noteworthy obstruction to everyday activities. Essentially, SPD represents a failure to modulate the effects of incoming \\ Springer Open}


sensory inputs; this raises the issue of whether the integration of inputs across sensory systems is functioning appropriately in this population. The principal function of the multisensory integration system is to combine the signals that enter the brain through the different sensory epithelia so that the many forms of energy produced by the same object or event will be treated as a single entity. It helps to simplify the world and leads to significant behavioral efficiency gains [8].

The literature shows a connection between processing challenges and sensory integration and performance of activities of daily living (ADLs) such as sleeping, dressing, eating, playing, and taking part in leisure and school-related activities [9]. According to Brown and Dunn, "areas of concern emerge only when a person's sensory processing pattern seems incompatible with his or her desired or necessary life activity choices" [7]. Motor system development and motor planning partially rely on sensory input which offers feedback and guides motor function so that potential motor errors can be avoided [10].

Although sensory-based disorders as impaired tactile, proprioceptive, kinesthetic, and pain perception are not commonly thought of as a primary feature of $\mathrm{CP}$, they occur frequently in this population and remain an under-studied and under-addressed area [10-12]. Further studies are recommended to evaluate sensory processing in children with CP. This exploration remains a vast and promising field of research to be pursued [12]. Most studies addressing sensory processing in children with $\mathrm{CP}$ use sophisticated neuroimaging equipment, which is not widely available in clinical settings. Using clinical measures to assess sensory processing in children with CP is still not common [13].

The Sensory Integration and Praxis Test (SIPT) is regarded as the "gold standard" test for detecting sensory discrimination and sensorimotor abnormalities [6]. However, it does not measure sensory modulation [14]. Sensory modulation is typically measured by the Sensory Profile (which has two versions) and the Sensory Processing Measure [6, 14].

Sensory profile 2 (SP2) is a standardized test. The advantages of SP2 are that it is simple to use, quick to administer, is evidence, theory, and strength based. SP2 is family and child centered and also contextually relevant. It provides a comprehensive method to capture the child's responses to sensory experiences in natural settings. It measures current performance and can be reused to measure differences across time and intervention. Additionally, it addresses familiar activities and behaviors to caregivers and helps them be more engaged in assessment and the discussion of the treatment plan [15].

The SP2 has some disadvantages. For starters, it relies on a caregiver's ability to report on their child's functioning, which may lead to responder bias. There may also be inconsistencies between the responses of the caregiver and the observations of the therapist. Moreover, despite its ability to detect the presence of modulation problems, it is unable to thoroughly determine the presence of sensory discrimination problems [16]. Furthermore, the SP2 is yet to be validated in the Egyptian population. Finally, it has not been translated into Arabic.

Knowledge about a person's patterns of sensory processing may contribute to the design of more effective interventions and the advancement of knowledge. When people have a vision about the reasons behind challenging situations, they can generalize the information to other circumstances and make better judgments and choices that make life easier [17].

This area of knowledge is also supposed to generate more research questions aiming for a wider look at disabled children's needs and how to deal with them.

Therefore, this study aims to analyze sensory systems processing in children with SCP (GMFCS levels I, II, and III), using Child Sensory Profile 2 (CSP-2).

\section{Methods \\ Study design}

A prospective cross-sectional study design has been performed. The study agreed with the STROBE statement [18]. The study was approved by the Ethical Committee under the code (P.T.REC/012/003055).

\section{Participants and procedures}

Twenty SCP children were recruited from several rehabilitation centers specialized in children's care. In order to recruit the sample, rehabilitation centers were contacted about the purpose of the study and the targeted criteria for children to participate. Meetings were set with caregivers to explain the study rationale and explain how to fill the CSP-2 questionnaires. Those who agreed to participate and met the inclusion criteria signed informed consent before taking place in the study. All questions by participating caregivers were answered before, during, and after filling the questionnaires. Results were verbally conducted to caregivers, and suggestions on how to deal with children accordingly were provided. The recruitment and assessment took place between February 2021 and June 2021.

This study was narrowed down to focus on SCP. According to previous studies $[19,20]$, the better the gross motor capabilities are, the finer the sensory processing capacities. Therefore, we concentrated on children with higher gross motor abilities of Gross Motor Function Classification System (GMFCS) levels I, II, and III so that we may assume that their sensory experiences are less limited by their motor abilities. 
We included children of both sexes, aged between 6 and 9 years, and whose caregivers are of a good command of the English language.

We excluded (1) children with severe comorbidities besides CP (i.e., diabetes, myopathy, neuropathy, and uncontrolled epilepsy), burn injuries, hearing or vision loss; to reduce factors that may compromise their sensory experiences. (2) Children with autism; since autism has been-supported by several studies-associated with SPD.

\section{Variables and data measurements}

The primary variable in this study was the reported sensory system section, measured by CSP-2. CSP-2 lies under the umbrella of SP2. The Sensory Profile 2 is the newest version of the Sensory Profile developed by Winnie Dunn; it is a set of standardized caregiver questionnaires consisting of the Infant SP2 (birth-to-6 months), Toddler SP2 (7-to-35 months), Child SP2 (3-to-15 years old), and Short SP2 (3-to-15 years old). In addition to School companion SP2, which is a teacher questionnaire for students aged from 3 to 14 years old [15].

The parent/caregiver complete the SP2 questionnaire according to the frequency of the child's responses to various sensory experiences using a five-point scale $(1=$ almost always, $2=$ frequently, $3=$ half of the time, $4=$ occasionally, $5=$ almost never, or $0=$ does not apply). Does not apply should be used exceptionally when the question is not relevant to the child, or when the parent/ caregiver has never observed the behavior. The advantages of SP2 are that it is simple to use and quick to administer and interpret. Furthermore, there are no double negatives in the SP2, which makes it easier to read than the SP. CSP-2 is an 86-item caregiver questionnaire that covers a wide age range from 3:00 to 14:11 years old [15].
Dunn developed a Normal Curve and SP2 Classification System based on responses from a normative sample of children without disabilities. Based on a bell curve normed distribution, the raw score total for each quadrant can be classified as "much less than others," "less than others," "just like the majority of others," "more than others," and "much more than others" [21].

Figure 1 shows the normal curve and the SP2 classification system provided by Dunn [15].

The items of CSP-2 come in three groups which are sensory processing patterns (i.e., the quadrants), sensory systems, and behavior associated with sensory processing. Sensory System Scores (Sensory Sections) subsets include Auditory Processing: which measures the person's response to things heard; Visual Processing: which measures the person's response to things seen; Touch (Somatosensory) Processing: which measures the person's response to stimuli that touch the skin; Movement (Vestibular) Processing: which measures the person's response to movement; Body Position (Proprioceptive) Processing: which measures the person's response to changes in joint and muscle positions; and Oral Sensory Processing: which measures the person's response to touch and taste in the mouth [15].

The test-retest reliability is good to excellent. Most of the inter-rater reliability coefficients were acceptable to good. The coefficients in the visual and touch processing areas were lower. The validity correlations between the SP and SP2 were moderate to high $[15,16]$. Sensory Profile 2 shows good construct validity [22].

\section{Statistical methods}

Descriptive results were generated by calculating the means and standard deviation. The results were compared to the normative data provided by the work of Dunn in the SP2 user's manual [15].

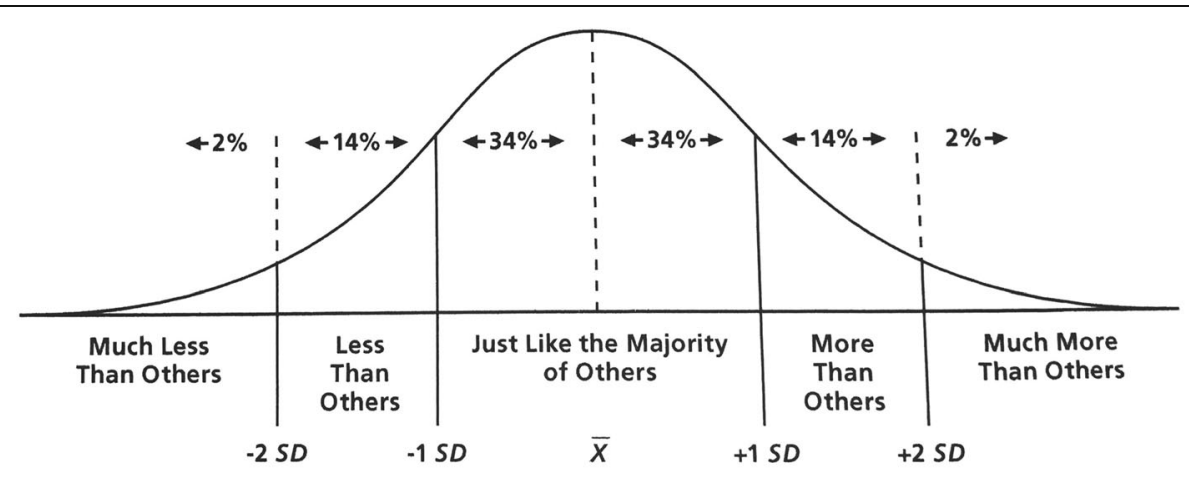

Low Score

High Score

Fig. 1 The normal curve and the sensory profile 2 classification system (Dunn 2014) 
Table 1 The distribution of the SCP children according to SCP subtypes, gender, and the GMFCS levels

\begin{tabular}{|c|c|c|c|c|c|c|}
\hline Subtype & Gender & 1 & II & III & Total & Percent \% \\
\hline \multirow[t]{2}{*}{ Unilateral } & Male & 3 & 0 & 0 & 3 & $60 \%$ \\
\hline & Female & 1 & 1 & 0 & 2 & $40 \%$ \\
\hline \multirow[t]{2}{*}{ Bilateral } & Male & 1 & 4 & 7 & 12 & $80 \%$ \\
\hline & Female & 0 & 2 & 1 & 3 & $20 \%$ \\
\hline
\end{tabular}

\section{Results}

\section{Characteristics of the sample (demographic data)}

A total of 20 children with a mean age of 7.25 (SD \pm 1.019545823) participated in this study.

The majority of the sample (65\% of the sample) were in the younger group $(n=13)$ and the minority $(35 \%$ of the sample) were in the older group $(n=7)$.

$75 \%$ of the participants were male $(n=15)$ and $25 \%$ were female $(n=5)$

\section{Classification of participants}

Twenty-five percent of participants were classified as unilateral SCP, and 75\% were classified as bilateral.

Twenty-five percent of participants were classified according to GMFCS as level I, 35\% were classified as level II, and $40 \%$ were classified as level I.

Table 1 shows the distribution of the children with SCP according to SCP subtypes, gender, and the GMFC S levels.

\section{Distribution of all children with SCP according to their response to sensory systems}

Table 2 shows the percentage distribution for the six sensory system sections. The highest percentage of participants scoring in the collective "More Group" was found in the "Body Position" section (95\%), with "much more" representing the majority by $70 \%$; followed by the "Movement" section (65\%) with "More than" representing the majority by $40 \%$.

The participants mostly scored "just like" in the other four sections as follows: "Oral sensory" and "Visual" by $80 \%$, "Auditory" by $70 \%$, and "Touch" by $60 \%$. No participants scored in any section as "Less than" or "Much Less than."

There was no single participant found to score out of the norm in all six sections. Thirty percent of the participants faced sensory system difficulties in only one section, while $35 \%$ of them faced sensory system difficulties in 2 to 3 sections, and $35 \%$ faced difficulties in 4 to 5 sections.

\section{Sensory processing in the sensory systems in both types} of SCP

Figure 2 shows a box plot chart that was made using raw scores to form a visual representation of all data collected from all participants in all sensory systems sections; it shows that the data set has a symmetrical distribution since the mean and the median are close together. Touch has the most variable data for it has the highest range.

Means of auditory (20.25), visual (14.95), touch (20.3), and oral sensory (20.8) are below the dashed line which refers to the line between "just like" and "more than" which means that they tend to be within normal range. While means of movement (21.1) and body position (24.6) lie above the dashed line, which means they fall out of the normal range.

\section{Sensory processing in each of the six sections of} sensory systems in both types of SCP (unilateral and bilateral)

Figure 3 shows the box plots of the auditory processing section. Bilateral SCP has a longer box which means more dispersed data. The mean of both types are very similar (20.2) and they both lie under the dashed line which means they both tend to fall within the normal range and score as "just like."

Figure 4 shows the box plots of the visual processing section. The means of both types are very similar (15) for unilateral and (14.93) for bilateral. They both lie under the dashed line, which means they both

Table 2 Distribution of All children with SCP according to their response to sensory systems

\begin{tabular}{lllllll}
\hline & Auditory & Visual & Touch & Movement & Body position & Oral sensory \\
\hline Much more & $0 \%$ & $5 \%$ & $15 \%$ & $25 \%$ & $70 \%$ & $5 \%$ \\
More than & $30 \%$ & $15 \%$ & $25 \%$ & $40 \%$ & $25 \%$ & $15 \%$ \\
More Group & $30 \%$ & $20 \%$ & $40 \%$ & $65 \%$ & $95 \%$ & $20 \%$ \\
Just like & $70 \%$ & $80 \%$ & $60 \%$ & $35 \%$ & $5 \%$ & $80 \%$ \\
\hline
\end{tabular}


Auditory $\square$ Visual $\square$ Touch $\square$ Movement $\square$ Body Position $\square$ Oral sensory

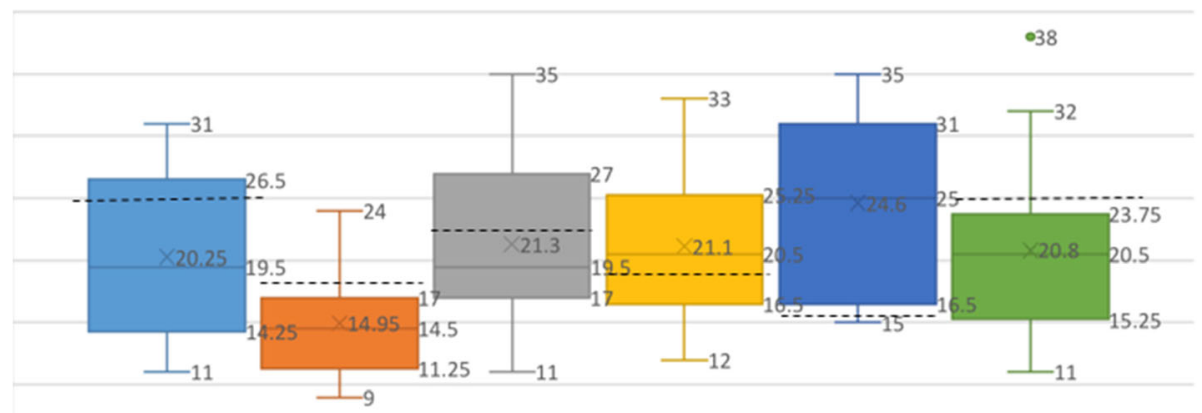

Fig. 2 Box plot showing sensory processing in the sensory systems in children with SCP

tend to fall within the normal range and score as "just like."

Figure 5 shows the box plots of the touch processing section. The means of both types are similar (20.6) for unilateral and (21.53) for bilateral. They both lie under the dashed line which means they both tend to fall within the normal range and score as "just like"; however, bilateral mean lies so close to the dashed line; therefore, it lies on edge.

Figure 6 shows the box plots of movement processing results. The means of both types are very similar (21.2) for unilateral and (21.07) for bilateral. They both lie above the dashed line, which means they both tend to fall outside the normal range.

Figure 7 shows the box plots of the body position processing section. The mean of unilateral is (19.2) which is far from the median; the median of the unilateral lies outside the bilateral box which indicates the presence of a significant difference between the two groups. As for the bilateral mean (26.4), it is similar to the median. They both lie above the dashed line, which means they both fall outside the normal range.

Figure 8 shows the box plots of the oral sensory processing section. The mean of both types is similar (20.4) for unilateral and (20.93) for bilateral. They both lie under the dashed line which means they both tend to fall within the normal range and score as "just like."

\section{Comparison between sensory sections mean scores in children with unilateral and bilateral SCP}

Table 3 represents a summary comparison between mean scores of children with unilateral and bilateral SCP. The means of all sections were very similar except for the "body position" section, where unilateral was nearer to the "just like" range, which is from 5 to 15 of the raw score.

\section{Unilateral $\square$ Bilateral}

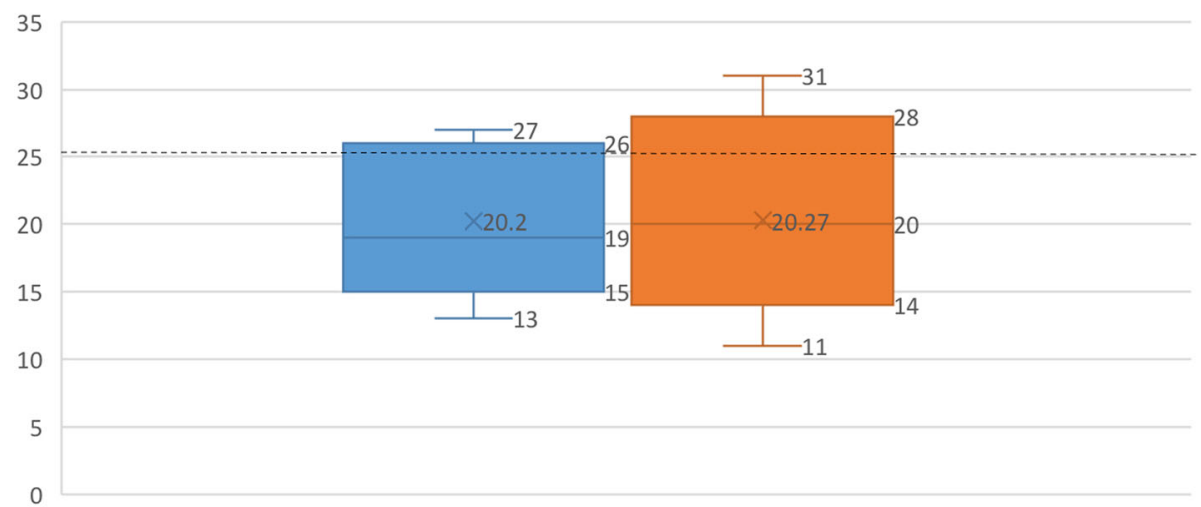

Fig. 3 Box plots of the auditory processing section in children with unilateral and bilateral SCP 
Unilateral $\square$ Bilateral

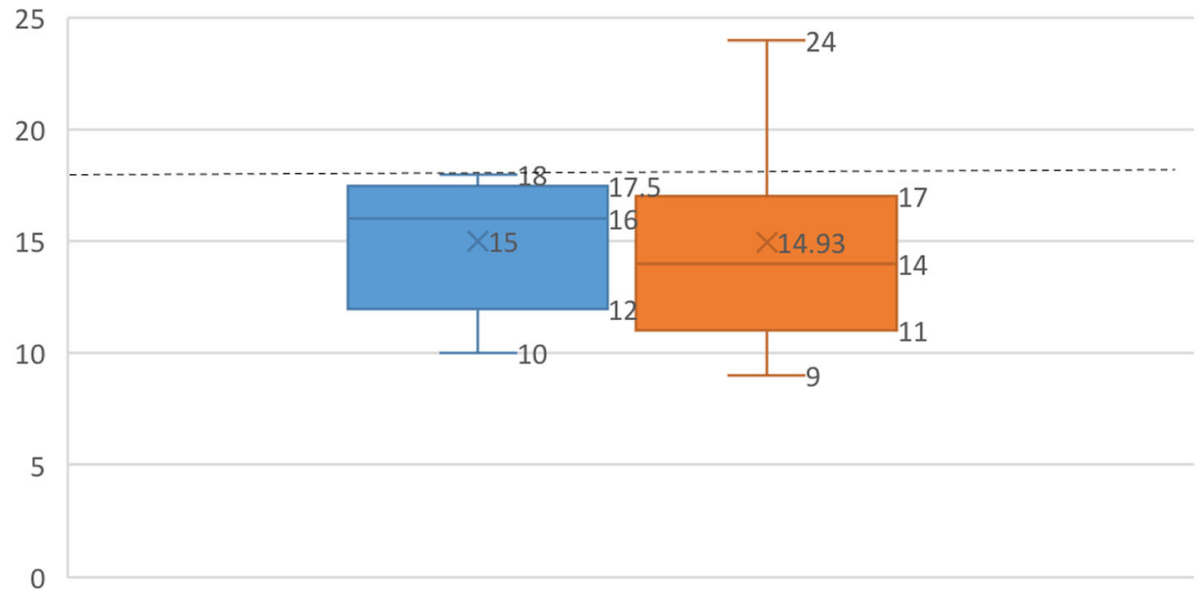

Fig. 4 Box plots of the visual processing section in children with unilateral and bilateral SCP

Distribution of children of each group ( Unilateral and Bilateral SCP) in the sensory systems

Table 4 shows the distribution of children of each group (unilateral and bilateral SCP) in the sensory systems.

In children with unilateral SCP The majority scored as "just like" in auditory by $60 \%$, visual by $80 \%$, touch by $80 \%$, and oral sensory by $80 \%$; while in movement and body position, they scored in the collective more group by $60 \%$ and $80 \%$ respectively.
In children with bilateral SCP The majority scored as "just like" in auditory by $73.33 \%$, visual by $80 \%$, touch by $53.33 \%$, and oral sensory by $80 \%$; while in movement, they scored in the collective more group by $66.67 \%$. All participants with bilateral SCP scored in the collective more group.

\section{Discussion}

This study aims to investigate the processing of sensory systems in children with SCP (GMFCS levels I, II, and III) using CSP-2.

Unilateral $\square$ Bilateral

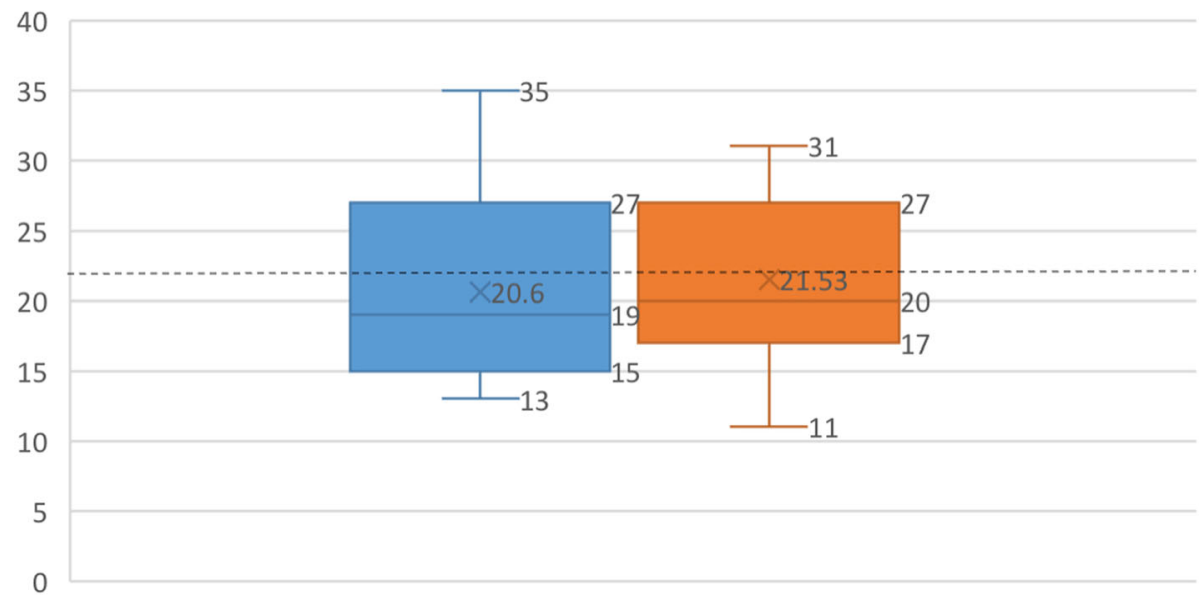

Fig. 5 Box plots of the touch processing section in children with unilateral and bilateral SCP 
Unilateral $\square$ Bilateral

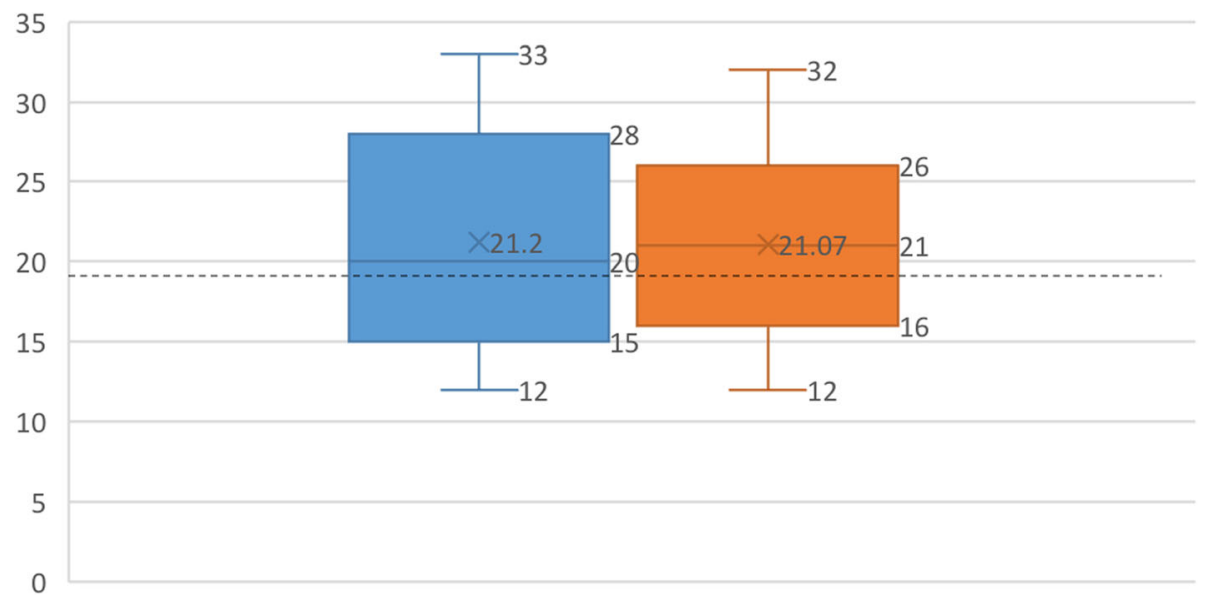

Fig. 6 Box plots of the movement processing section in children with unilateral and bilateral SCP

Sensory-based disorders were found to frequently occur in those children [23, 24]. However, the study of sensory processing and integration in this population is promising [12, 25].

Our results showed that children with SCP face processing difficulties regarding sensory systems. The majority of SCP children present greater problems with body position (proprioceptive) and movement (vestibular) processing than other sensory systems compared to the normative data provided by Dunn; which matches the results of previous studies done on more types of $\mathrm{CP}[16,26]$ or a wider range of GMFCS levels [25].

Vestibular processing and proprioceptive processing deficits may be a reason for balance impairment in addition to poor muscle control, which magnifies the problem [16, 27]. The reason might be due to diminished thalamo-cortical projections from the thalamus to S1, which causes defects to somatosensory processing in $\mathrm{CP}$ patients [10].

Unilateral $\square$ Bilateral

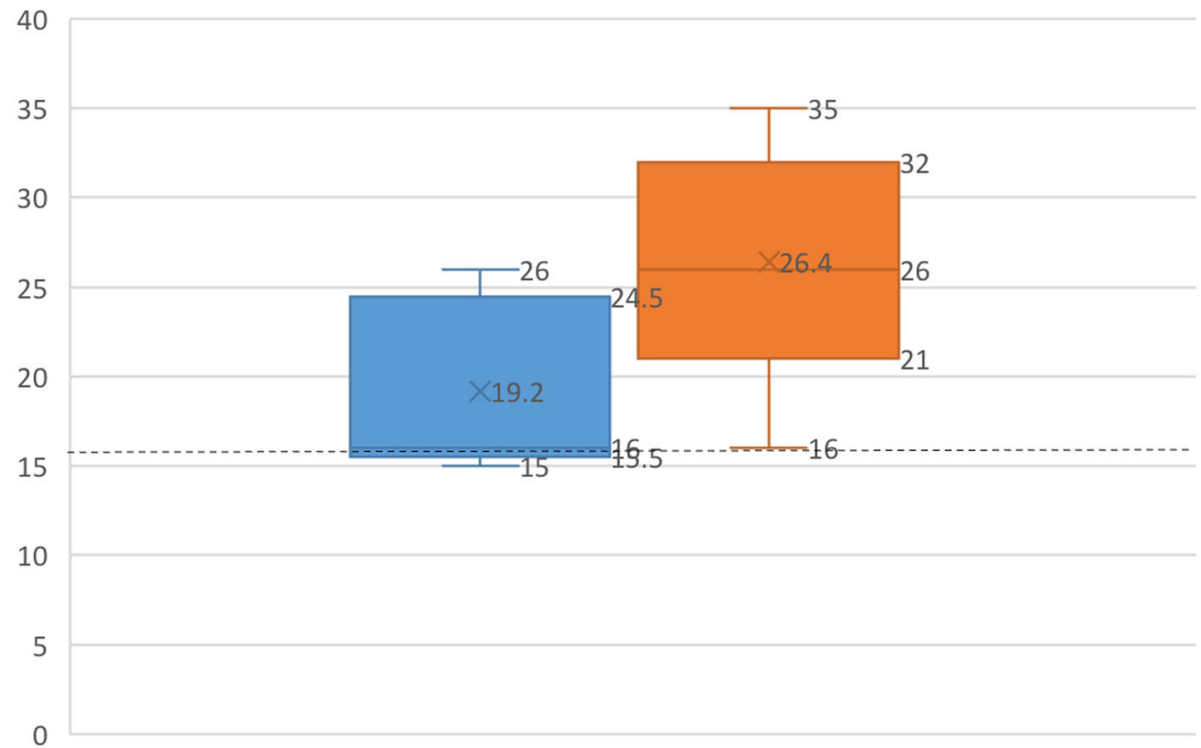

Fig. 7 Box plots of body position processing in children with unilateral and bilateral SCP 
Unilateral $\square$ Bilateral

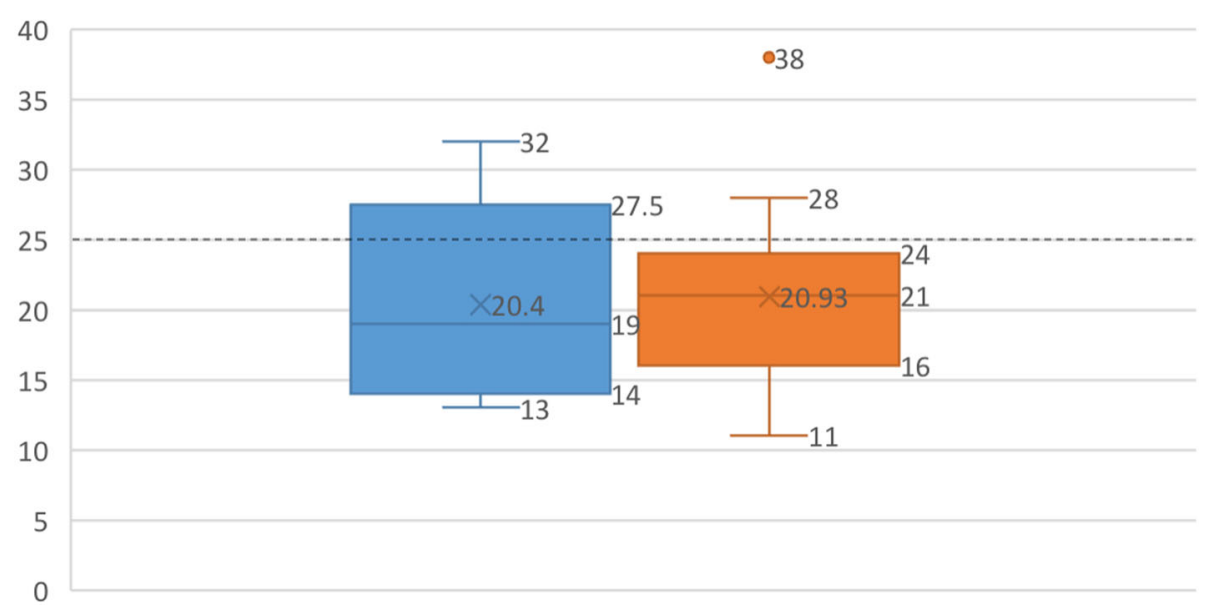

Fig. 8 Box plots of the oral sensory processing section in children with unilateral and bilateral SCP

Touch processing came in third place for $40 \%$ of children presented with difficulties in touch processing. Tactile processing issues vary dramatically among cerebral palsy subtypes, according to research [26]. According to Pavaõ [25], tactile processing may also be due to structural deficits.

Thirty percent of the children showed auditory processing difficulties. There was no difference between children with unilateral and bilateral SCP groups in the mean scores, and both fell in the "just like" zone. These results partially confirm some previous studies [16, 20, 25] while not confirming at all to others [26], but we must put into consideration that there are noticeable differences in the inclusion criteria.

It was also revealed that they struggle the least with oral sensory and visual information, although some difficulties were present.

By comparing raw scores of children with unilateral and bilateral SCP, it was found that the only section that showed a significant difference between the two categories is "body position" (proprioceptive). This

Table 3 Comparison between mean scores of sensory sections in children with unilateral and bilateral SCP

\begin{tabular}{lll}
\hline & Unilateral & Bilateral \\
\hline Auditory & 20.2 & 20.27 \\
Visual & 15 & 14.93 \\
Touch & 20.6 & 21.33 \\
Movement & 21.2 & 21.07 \\
Body position & 19.2 & 26.4 \\
Oral sensory & 20.4 & 20.93 \\
\hline
\end{tabular}

shows that children with bilateral SCP convey proprioceptive processing problems more than children with unilateral SCP. Proprioceptive problems in the unilateral population may be due to uneven experiences they are exposed to from crawling through to walking [16].

Unlike what we have found, previous research found that oral sensory processing was significant in children with unilateral SCP [20]. The difference may be due to the small sample size of the current preliminary study or the difference in population culturally or geographically.

The presence of more visual processing difficulties in children with unilateral SCP has been reported in a previous study [16], while another study reported similar results to this study [20].

This study was limited by:

1- Small sample size

2- Being confined to caregivers who are of a good command of the English language, which was hard and time-consuming to recruit in a population where Arabic is the mother tongue.

\section{Conclusions}

According to our findings, children with SCP levels I, II, and III on GMFCS show processing difficulties concerning sensory systems compared to normative data. The main difficulties manifested are concerning body position (proprioceptive) and movement (vestibular). Knowing this valuable information allows us a better understanding of their sensory experiences and their processing struggles that might affect the 
Table 4 Distribution of children of each group (unilateral and bilateral SCP) in the sensory systems

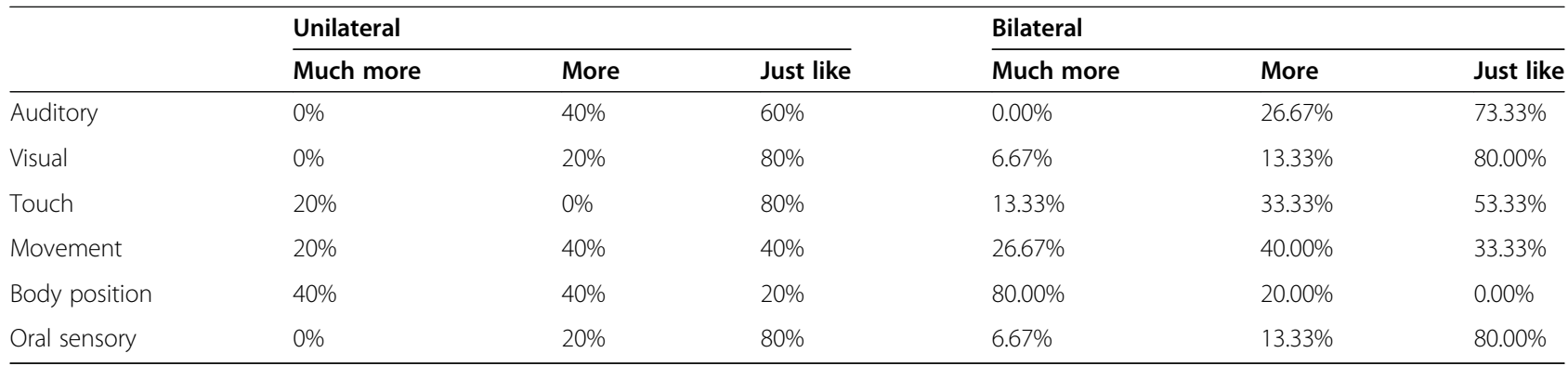

way they deal with their environment, and consequently enables therapists and caregivers to actively participate in improving their treatment plans and how to deal with them more efficiently.

\section{Abbreviations}

ADLs: Activities of daily living; CP: Cerebral palsy; CSP-2: Child Sensory Profile 2; GMFCS: Gross Motor Function Classification System; SPD: Sensory processing disorder; SP: Sensory Profile; SP2: Sensory Profile 2; SCP: Spastic CP; SIPT: The Sensory Integration and Praxis Test

\section{Acknowledgements}

The authors want to acknowledge the efforts of the rehabilitation centers and caregivers who co-operated and made this study possible.

\section{Authors' contributions}

BM suggested the research idea, helped in data collection, and analyzed and interpreted the data. She is the major contributor in the writing process. EG and AF revised the data analysis, and they were contributors in the writing process. AF was the main supervisor of the work in this study. She contributed in conception of the research idea and design of the work. All authors read and approved the final version of the manuscript.

\section{Funding}

This research received no external funding.

\section{Availability of data and materials}

Data will be shared on specific request to the corresponding author depending upon the nature and purpose of the requirement.

\section{Declarations}

\section{Ethics approval and consent to participate}

This study was approved by the ethics committee of Faculty of physical therapy, Cairo University with approval number [P.T.REC/012/003055]. The caregivers read and signed a written informed consent form.

\section{Consent for publication}

Not applicable.

\section{Competing interests}

The authors declare that they have no competing interests.

\section{Received: 8 July 2021 Accepted: 24 August 2021}

\section{Published online: 16 December 2021}

\section{References}

1. Bartels EM, Korbo L, Harrison AP. Novel insights into cerebral palsy. J Muscle Res Cell Motil. 2020;41(2):265-7 Available from: https://doi.org/10.1007/s1 0974-020-09577-4

2. Van Eyk CL, Corbett MA, Gardner A, Van Bon BW, Broadbent JL, Harper K, et al. Analysis of 182 cerebral palsy transcriptomes points to dysregulation of trophic signalling pathways and overlap with autism. Transl Psychiatry. 2018;8(1):1-10 Available from: https://doi.org/10.1038/s41398-018-0136-4.
3. Ozkan Y. Child's quality of life and mother's burden in spastic cerebral palsy: a topographical classification perspective. J Int Med Res. 2018;46(8):3131-7. https://doi.org/10.1177/0300060518772758.

4. Cans C. Surveillance of cerebral palsy in Europe: a collaboration of cerebral palsy surveys and registers. Dev Med Child Neurol. 2000;42(12):816-24.

5. Patel DR, Neelakantan M, Pandher K, Merrick J. Cerebral palsy in children: a clinical overview. Transl Pediatr. 2020;9(1):S125-35. https://doi.org/10.21037/ tp.2020.01.01.

6. Jorquera-Cabrera S, Romero-Ayuso D, Rodriguez-Gil G, Triviño-Juárez JM. Assessment of sensory processing characteristics in children between 3 and 11 Years Old: a systematic review. Front Pediatr. 2017:5:57. https://doi.org/1 0.3389/fped.2017.00057. Erratum in: Front Pediatr. 2017;5:266

7. Metz AE, Boling D, DeVore A, Holladay H, Liao JF, Vlutch KV. Dunn's model of sensory processing: an investigation of the axes of the four-quadrant model in healthy adults. Brain Sci. 2019;9(2):35. https://doi.org/10.3390/bra insci9020035.

8. Foxe JJ, Del Bene VA, Ross LA, Ridgway EM, Francisco AA, Molholm S. Multisensory audiovisual processing in children with a sensory processing disorder (II): speech integration under noisy environmental conditions. Front Integr Neurosci. 2020;14(July):1-8.

9. Schaaf RC, Dumont RL, Arbesman M, May-Benson TA. Efficacy of occupational therapy using ayres sensory integration ${ }^{\oplus}$ : a systematic review. Am J Occup Ther. 2018;72(1):7201190010p1-7201190010p10. https://doi. org/10.5014/ajot.2018.028431.

10. Papadelis C, Ahtam B, Nazarova M, Nimec D, Snyder B, Grant PE, et al. Cortical somatosensory reorganization in children with spastic cerebral palsy: A multimodal neuroimaging study. Front Hum Neurosci. 2014; 8(September):1-15.

11. Newell A, Cherry S, Fraser M. Principles of rehabilitation: occupational and physical therapy. In: Orthopedic Care of Patients with Cerebral Palsy; 2020. p. 221-50.

12. Pavaõ SL, Silva FPDS, Savelsbergh GJP, Rocha NACF. Use of sensory information during postural control in children with cerebral palsy: Systematic review. J Mot Behav. 2015;47(4):291-301. https://doi.org/10.1080/ 00222895.2014.981498

13. Pavão SL, Lima CRG, Rocha NACF. Association between sensory processing and activity performance in children with cerebral palsy levels I-II on the gross motor function classification system. Brazilian J Phys Ther. 2020;(xx) Available from: https://doi.org/10.1016/j.bjpt.2020.05.007.

14. Mulligan S, Douglas S, Armstrong C. Characteristics of idiopathic sensory processing disorder in young children. Front Integr Neurosci. 2021;15(April):1-10.

15. Dunn W. Sensory profile 2 user's manual. Bloomington: Pearson; 2014.

16. Louwrens S. Sensory modulation patterns in children with cerebral palsy : a comaparative-descriptive study: University of Pretoria; 2018. Available from: http://hdl.handle.net/2263/67775. Accessed 25 Mar 2021.

17. Dunn W. The sensations of everyday life: Empirical, theoretical, and pragmatic considerations. Am J Occup Ther. 2001;55(6):608-20. https://doi. org/10.5014/ajot.55.6.608

18. von Elm E, Altman DG, Egger M, Pocock SJ, Gøtzsche PC, Vandenbroucke JP, STROBE Initiative. The Strengthening the Reporting of Observational Studies in Epidemiology (STROBE) statement: quidelines for reporting observational studies. J Clin Epidemiol. 2008;61(4):344-9. https://doi.org/10.1 016/j.jclinepi.2007.11.008.

19. Park M-O. The relationship between sensory processing abilities and gross and fine motor capabilities of children with cerebral palsy. J Korean Soc Phys Med. 2017;12(2):67-74. https://doi.org/10.13066/kspm.2017.12.2.67. 
20. Jovellar-Isiegas P, Collados IR, Jaén-Carrillo D, Roche-Seruendo LE, García CC. Sensory processing, functional performance and quality of life in unilateral cerebral palsy children: a cross-sectional study. Int J Environ Res Public Health. 2020:17(19):1-13.

21. Simpson K, Adams D, Alston-Knox C, Heussler HS, Keen D. Exploring the sensory profiles of children on the autism spectrum using the short sensory profile-2 (SSP-2). J Autism Dev Disord. 2019;49(5):2069-79 Available from: https://doi.org/10.1007/s10803-019-03889-2.

22. Dean E, Dunn W, Little L. Validity of the sensory profile 2: a confirmatory factor analysis. Am J Occup Ther. 2016;70(4_Supplement_1):7011500075p1 Available from: http://ajot.aota.org/article.aspx?doi=10.5014/ajot.2016.70S1PO7054.

23. O'Shea TM. Diagnosis, treatment, and prevention of cerebral palsy in nearterm/term infants. Clin Obstet Gynecol. 2008;51(4):816-28 Available from: http://www.ncbi.nlm.nih.gov/pmc/articles/pmc3051278/.

24. Bumin $\mathrm{G}$, Kayihan $\mathrm{H}$. Effectiveness of two different sensory-integration programmes for children with spastic diplegic cerebral palsy. Disabil Rehabil. 2001;23(9):394-9. https://doi.org/10.1080/09638280010008843.

25. Pavão SL, Adriana N, Ferreira C. Sensory processing disorders in children with cerebral palsy. Infant Behav Dev. 2017;46:1-6 Available from: https:// doi.org/10.1016/j.infbeh.2016.10.007.

26. Prakash AJA, Vaishampayan A. A preliminary study of the sensory processing abilities of children with cerebral palsy and typical children on the Sensory Profile. Indian J Occup Ther. 2007;XXXIX(2):27-34 Available from: https://www.aiota.org/temp/ijotpdf/ibat07i2p27.pdf.

27. Tramontano M, Medici A, losa M, Chiariotti A, Fusillo G, Manzari L, et al. The effect of vestibular stimulation on motor functions of children with cerebral palsy. Mot Control. 2017;21 (3):299-311 Available from: https://doi.org/1 0.1123/mc.2015-0089.

\section{Publisher's Note}

Springer Nature remains neutral with regard to jurisdictional claims in published maps and institutional affiliations.

\section{Submit your manuscript to a SpringerOpen ${ }^{\circ}$ journal and benefit from:}

- Convenient online submission

- Rigorous peer review

- Open access: articles freely available online

- High visibility within the field

- Retaining the copyright to your article

Submit your next manuscript at $\boldsymbol{\nabla}$ springeropen.com 